\title{
Contrast enhancement of soft tissues in Computed Tomography images
}

\author{
Roman Lerman, Daniela S. Raicu, Jacob D. Furst \\ Intelligent Multimedia Processing Laboratory \\ School of Computer Science, Telecommunications, and Information Systems \\ DePaul University, Chicago, IL 60604, USA
}

\begin{abstract}
*
Even though soft tissues are of primary interest to radiologists, they are represented using only $12.5 \%$ of the total number of gray levels in a typical DICOM format of a Computed Tomography (CT) scan. This poor distribution of gray levels reduces the overall contrast and the texture differences between individual organs, and poses a serious visualization problem since radiologists need clear visual representations of organs to produce proper diagnoses. In order to enhance the contrast within the soft tissues, the gray levels can be redistributed both linearly and nonlinearly using the gray level frequencies of the original CT scan. We propose a new nonlinear approach for contrast enhancement of soft tissues in CT images using both clipped binning and nonlinear binning based on a k-means clustering algorithm. The optimal number of bins, in particular the number of gray-levels, is chosen automatically using entropy and average distance between the histogram of the original gray-level distribution and the contrast enhancement function's curve. The contrast enhancement results were obtained and evaluated using $141 \mathrm{CT}$ images of the chest and abdomen from two normal CT studies.
\end{abstract}

Keywords: Contrast enhancement, visualization, soft tissues, computed tomography

\section{INTRODUCTION}

The purpose of this research is to propose a new approach for contrast enhancement of soft tissues in CT images and reduction of the redundancy in the image data. Redundant data is usually caused by large ranges of gray levels being used to represent images, which actually require significantly fewer gray levels. This concept is demonstrated in Figure 1 , in which an image is represented on the left-hand side and the gray levels used to produce that image are displayed on the right. Even though the image needs only two gray levels in order to capture its content, six gray levels are used to display it; therefore, instead of using only one bit to store the gray levels, 3 bits are used, requiring more storage space than needed. Furthermore, considering the DICOM format for CT images, 4096 gray levels are used to represent the content of a CT image, but in analyzing the CT images we noticed that only gray levels in the range of $(800,1400)$ are actually used to capture the soft tissues within these images. Figure 2 shows an example of a DICOM image for which even though the maximum gray level is 2398 , the number of gray-levels used to display the image is 4096 .

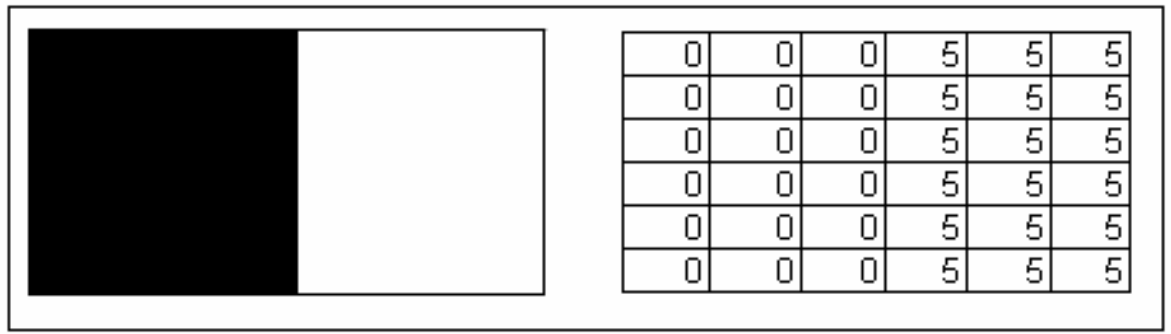

Figure 1: Original image and its gray level array representation

\footnotetext{
- This material is based upon work supported by the National Science Foundation under Grant No. 0453456.
} 
Abdomen CT 4096 Gray Levels

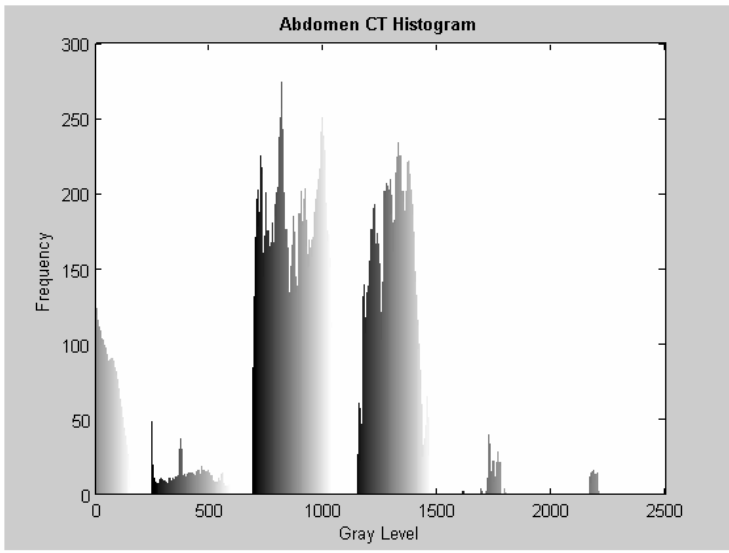

Figure 2: Figure 2: DICOM image and its histogram

\section{RELATED WORK}

There have been many methods developed for enhancing the features of interest in CT images. One such method is adaptive histogram equalization (AHE), which maps pixels of the original image to the resulting image so that the histogram of the resulting image shows a uniform distribution [5]. One possible drawback to this method is noise overenhancement and slow processing speeds, which has been addressed with updated methods such as the interpolated AHE [7]. Another technique is histogram transformation, which also attempts to flatten the histogram of the enhanced image using mappings based on local features. Sliding histograms on the other hand divide each image pixel into two components, one for texture and another for background. Weights are then applied to the two components in order to bring out features of interest. This technique however, requires much user intervention since the component weights are usually adjusted in order to find the visually optimal value [2]. Great strides in image contrast enhancement have also been made by popular software packages such as Photoshop. Photoshop has a feature called an unsharp mask, which actually enhances the contrast between tiny adjacent areas in an image. The feature actually allows the user to increase this type of contrast known as "local contrast" by a particular percentage.

\section{BINNING}

The proposed approach is a combination of clipped binning and nonlinear binning both based on a k-means clustering algorithm. The methods alter the amount and the distribution of gray levels in order to bring out the fine details of the image and to reduce noise. Since radiologists are heavily focused on examining the soft-tissues in CT images, two of the binning techniques utilize intensity windowing in order to focus in on the gray level interval which contains the softtissues.

\subsection{K-Means Clustering:}

K-Means clustering is a statistical technique used to establish the number of groups contained within a data set. In the beginning of the algorithm k-number of clusters are set and the data points move between those clusters such that the variability inside the clusters is minimized while the variability between the clusters is maximized [3].

\subsection{Clipped Linear}

Clipped binning uses the k-means algorithm to automatically determine the range of the gray levels for the soft tissues; regardless of the chosen number of clusters $(\mathrm{k})$ we found that the soft tissues are always placed in clusters found within the range of approximately 856 for the lower bound and 1368 for the upper bound. In general, for a clipped linear binning approach for soft tissues, all of the pixels in the lower bound are set to the lowest intensity (black), all of the pixels in the upper bound are set to the highest intensity (white), and all gray level values between the upper and lower 
bound are divided into equal bins. The original data was split up as follows: $[0,855]$ into bin $0,[856,1368]$ into bins $[1, \mathrm{k}]$, and $[1369,4095]$ into bin $\mathrm{k}+1$, with $\mathrm{k}$ being the chosen number of clusters.

\subsection{Nonlinear Binning}

In our proposed nonlinear binning approach, the results obtained from k-means clustering are used directly to form the bins in which to place the original gray levels. Figure 3 shows the steps involved in nonlinear binning: the histogram of the gray level data for the entire image collection is processed using the k-means algorithm and the similarities between the distributions of the individual gray levels across all images determine the clusters to be formed. The Euclidean distance was the similarity metric used in order to create the clusters. The mean of the points was used to determine the center of the cluster.

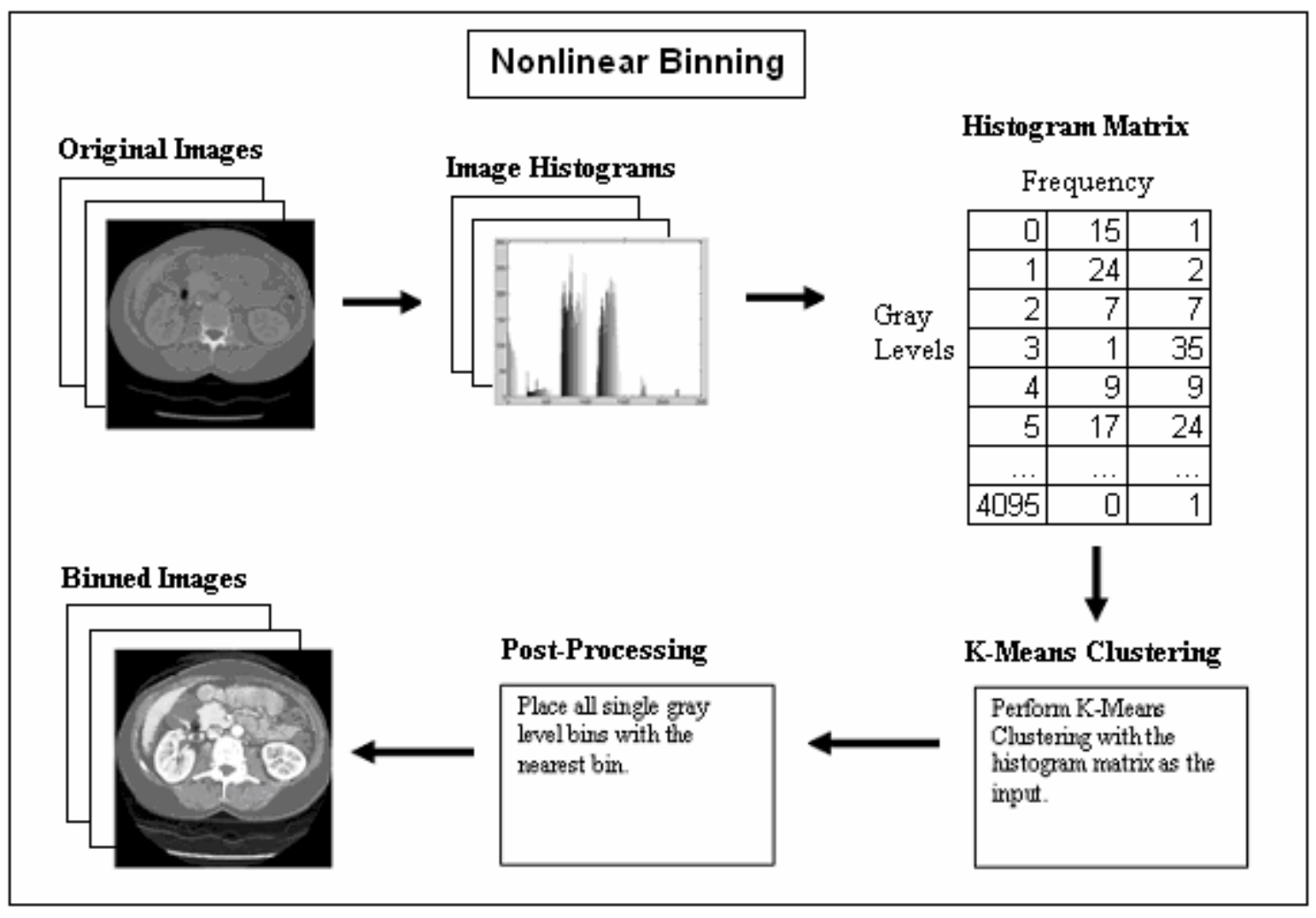

Figure 3: Diagram of nonlinear binning

\subsection{Clipped Nonlinear Binning}

The initial performance evaluation showed that the algorithm optimizes the images for viewing soft tissues, bony details or the entire image. Since we are interested only in the soft tissues, we combine the two approaches to produce clipped nonlinear binning: the pixels below the lower and above the upper bound are set to black, and the gray-levels between $(856,1368)$ are placed in bins using the nonlinear binning approach. Furthermore, bins that contain only one gray level are post-processed and merge with the most similar non-single value bins; this post processing step allows reduction of the different artifacts produced by the nonlinear binning. The original data was split up as follows $[0,855]$ into bin 0 , $[856,1368]$ into $\mathrm{k}$ bins (produced by clustering), and [1369,4095] into bin $\mathrm{k}+1$. 


\section{CRITERIA}

The optimization of clipped linear and clipped nonlinear binning is performed using the entropy criterion described as follows.

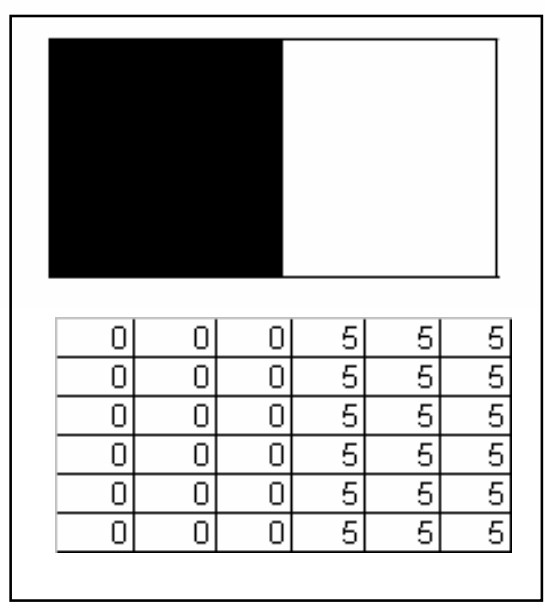

Figure 4: Image with gray level array representation

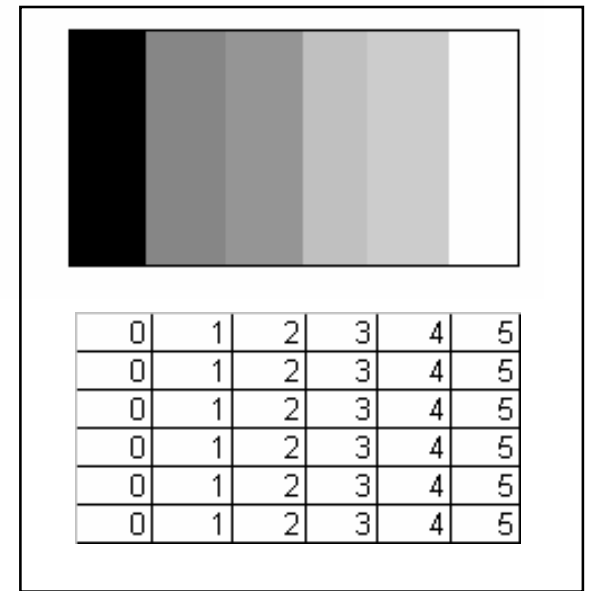

Figure 5: Image with gray level array representation

The gray level values of Figures 4 and 5 are represented by the charts directly below them. Both images have the same range of values so both are stored using six gray levels. It takes three bits to store each pixel in each image because there are six gray levels and 3 bits would allow the storage of up to eight gray levels. However, is it really necessary to use six gray levels for both images? The entropy value given by the formula,

$$
\text { Entropy }=-\sum_{i=1}^{n} p(i) \log _{2}(p(i)), \text { number of gray levels }
$$

will actually reveal the least number of bits with which a pixel can be stored so that all of the relevant information is retained. The $\mathrm{p}(\mathrm{i})$ in the formula stands for the probability of picking a particular gray level value out of the image at random.

So, for Figure 4 the entropy would be:

Entropy $=\left(-(1 / 2) \log _{2}(1 / 2)\right)+\left(-\log _{2}(1 / 2)\right)=1$.

For Figure 5 the entropy would be:

$$
\text { Entropy }=-6\left[(1 / 6) \log _{2}(1 / 6)\right]=2.58496 \text {. }
$$

This of course means that for figure 4 only $2^{1}$ or 2 gray levels are required in order to retain all of the relevant information, while for figure $5,2^{2.58496}$ or 6 gray levels are required [8].

The optimization using the entropy value is only effective when dealing with a good representation of the original image in which the area of interest is represented in a similar manner as in the original image. This is not possible with the nonlinear binning strategy since the binned images have only few gray levels. Therefore, in order to evaluate the nonlinear binning methodology, the mean normalized histogram for the entire image dataset and the curve of the nonlinear binning function are used (Figure 6): the Euclidean distance is then taken between the mean histogram and the curve for each point in the soft-tissue interval and this value is then averaged. This is done for different gray level values which result from the binning process and the gray level value for which the computed distance is smallest is considered to give the optimal number of gray level values - the one that optimizes contrast and minimizes information redundancy. Table 1 summarizes the three binning techniques with respect to their implementation and results evaluation. 


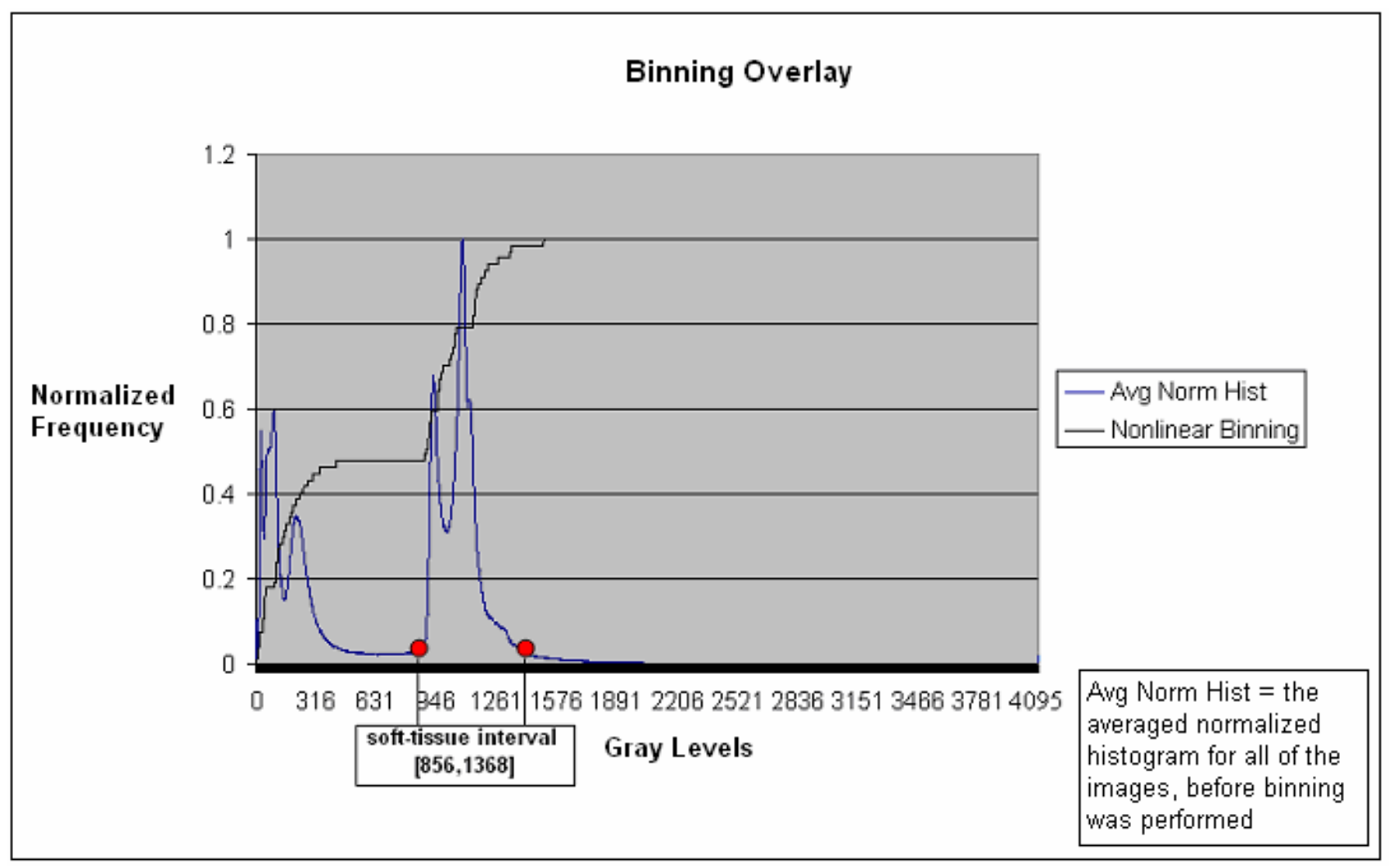

Figure 6: The average normalized histogram and the curve for the nonlinear binning function

\begin{tabular}{|c|c|c|c|}
\hline & Clipped Linear Binning & Nonlinear Binning & Clipped Nonlinear Binning \\
\hline Description & $\begin{array}{l}\text { * gray levels } 0-855 \text { bin to } 0 \\
* \text { gray levels } 856-1360 \text { divide equally } \\
\text { * gray levels } 1369-4095 \text { bin to maximum } \\
\text { gray level value }\end{array}$ & $\begin{array}{l}\text { * Perform K-Means Clustering } \\
\text { on the image histograms, apply } \\
\text { post-processing and perform } \\
\text { binning based on runs of gray } \\
\text { levels from the same cluster } \\
\end{array}$ & $\begin{array}{l}\text { * gray levels } 0-855 \text { bin to } 0 \\
\text { * gray lewels } 856-1360 \text { perform } \\
\text { nonlinear binning } \\
\text { * gray levels } 1369-4096 \text { bin to } \\
\text { maximum gray level value }\end{array}$ \\
\hline Evaluation & * Entropy & $\begin{array}{l}\text { * Awerage distance from the } \\
\text { mean normalized histogram to } \\
\text { the nomalized nonlinear binning } \\
\text { curve }\end{array}$ & * Entropy \\
\hline
\end{tabular}

Table 1: Overview of the three binning techniques considered in the proposed approach

\section{RESULTS}

Our preliminary results were obtained on 141 2D DICOM consecutive slices from 2 normal CT studies, each slice being of size 512 by 512 and having 12-bit gray level resolution. The maximum entropy value for clipped linear binning was 7.493, so the optimal number of gray levels is 256 , for that particular method. The maximum entropy value for clipped nonlinear binning was 7.5 so the optimal number of gray levels for this particular method is 256 . Since, the number of gray levels obtained for images binned using the clipped nonlinear methodology is controlled by the K-Means algorithm, the optimal value was judged to be the closest one given by the K-Means algorithm, which was 263 . The optimal 
number of bins for nonlinear binning was judged to be the number of bins which minimized the Euclidean distance between the overall histogram for the image set and the binning function; 65 gray levels was found to be optimal with this technique. This number of gray levels minimized the function to histogram distance with a distance of .7483. Figure 7 shows several examples of binned images using the three techniques for the corresponding optimal number of gray-levels. It can be noticed that using clipped nonlinear binning the contrast of the soft tissues is significantly improved. The results were obtained using Matlab software and a GUI was created to allow the user to select an image and then automatically enhance the contrast using either nonlinear or clipped nonlinear binning technique as shown in Figure 8.

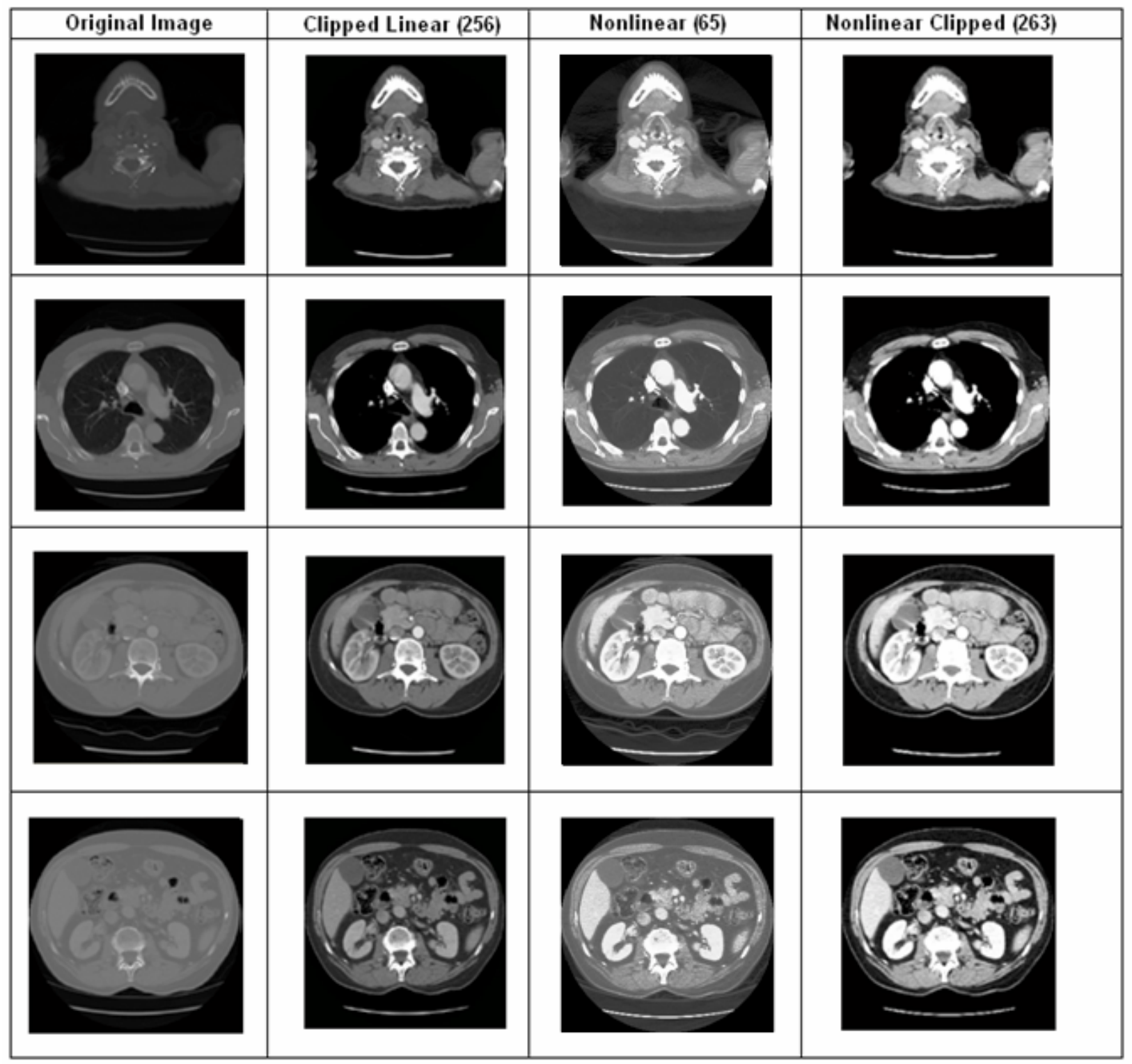

Figure 8: Results for contrast enhancement using the three binning approaches 


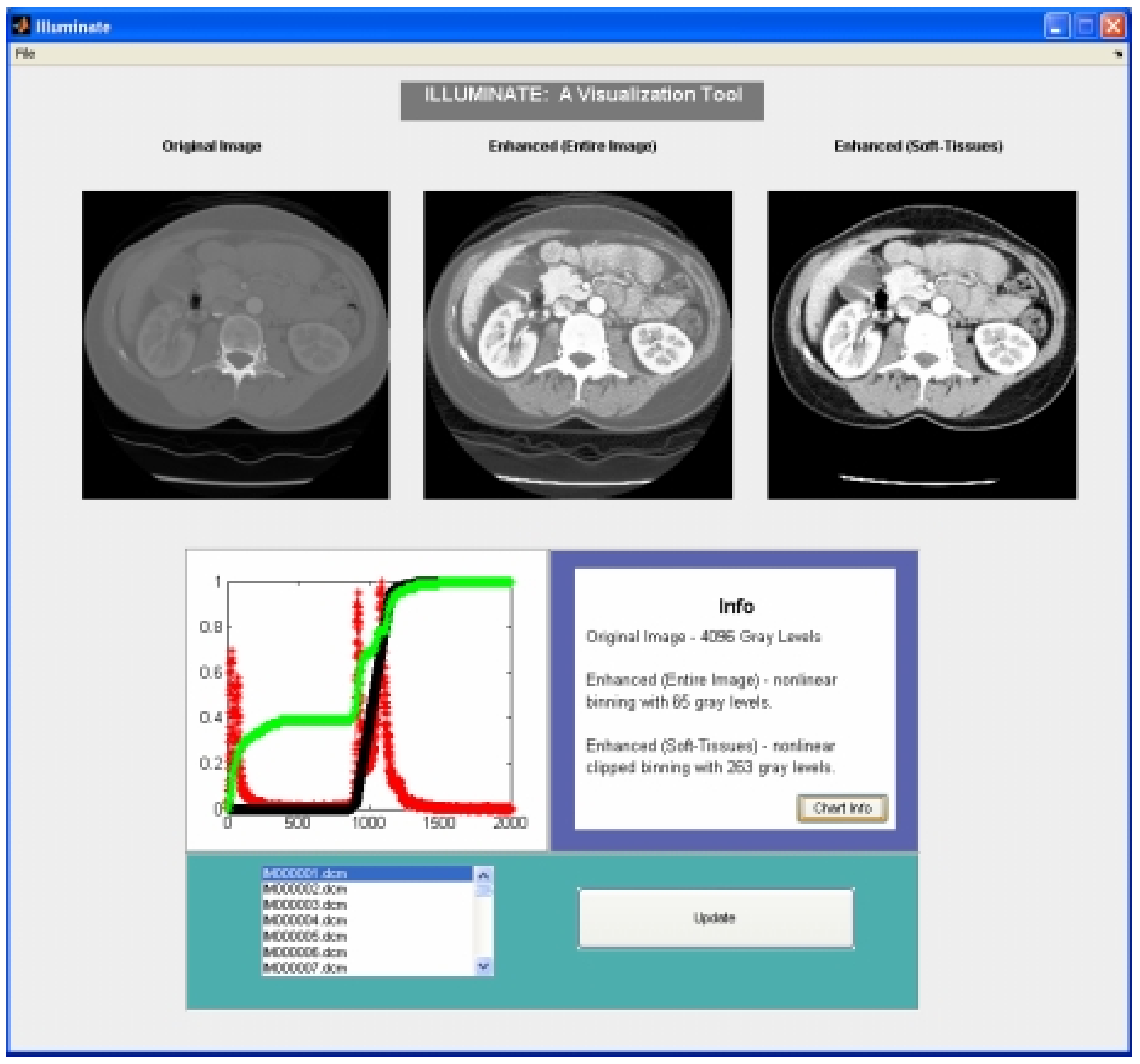

Figure 8: Screenshot of the visualization tool that uses nonlinear clipped binning for contrast-enhancement of soft tissues.

\section{CONCLUSIONS}

The contrast enhancement techniques and their respective optimizations can be very useful in several different areas of image processing. In addition to better visualization, the proposed binning strategies can be used as pre-processing steps when doing co-occurrence-based texture segmentation and classification of soft tissues in DICOM CT images of the chest and of the abdomen. 


\section{FUTURE WORK}

Although this research does delve deep into contrast enhancement using binning techniques there are other areas to be explored. The DICOM image sample set that was used for this research consisted of chest and abdomen CT scans, to be more complete in researching medical image enhancement, other types of CT scans could be used. The nonlinear

binning technique could also be tested with many different parameters. A different type of measure of central tendency could be used in the K-Means Clustering algorithm used in nonlinear binning.

\section{REFERENCES}

1. Barr, Donald R, "Measuring Information Gain In Tactical Operations" Operations Research Center of Excellence, 1996.

2. Chen, Kuei-Chun, "Color Image Enhancement", ACM, 1992.

3. Cluster Analysis, (2005. June 3), StatSoft. http://www.statsoft.com/textbook/stathome.html.

4. Gonzalez, R., Woods, R, Digital Image Processing, Prentice-Hall, Inc, Upper Saddle River, 2002.

5. Owens, Robyn, Spatial Domain Methods, (2005. July 15), http://homepages.inf.ed.ac.uk/rbf/CVonline/LOCAL_COPIES/OWENS/LECT5/node3.html.

6. Mathworld, (2005. July 19), http://mathworld.wolfram.com/.

7. Pizer, Stephen M. Adaptive Histogram Equalization and Its Variations. Computer Vision, Graphics, and Image Processing.

8. Sonka, M., Hlavac, V., Boyle R, Image Processing, Analysis, and Machine Vision, Brooks/Cole Publishing Company, Pacific Grove, 1999. 

